\title{
Effects of carvedilol reduce conjunctivitis through changes in inflammation, NGF and VEGF levels in a rat model
}

\author{
YING CHEN and XIANFEI HONG
}

\begin{abstract}
Department of Ophthalmology, Jinhua Traditional Chinese Medical Hospital, Jinhua, Zhejiang 321000, P.R. China
\end{abstract}
Received April 6, 2015; Accepted January 15, 2016

DOI: $10.3892 /$ etm.2016.3140

\begin{abstract}
Carvedilol is a novel third generation $\beta$-blocker that acts as an antagonist of $\beta$ and $\alpha$ adrenergic receptors, and is able to regulate various cell factors. In addition, it possesses antioxidant activity, is capable of reversing cardiac remodeling effects and has anti-arrhythmic effects. The present study aimed to investigate whether the effects of carvedilol were able to reduce conjunctivitis clinical scores. Initially, 24 Sprague Dawley (SD) rats were randomly divided into three equal groups as follows: Control group, model group and carvedilol group. The model and carvedilol group adult SD rats were injected with lipopolysaccharide (LPS) to induce conjunctivitis. In the carvedilol group, the eight SD rats with LPS-induced conjunctivitis also received $50 \mathrm{mg} / \mathrm{kg} / \mathrm{day}$ of carvedilol for 4 weeks. Next, the effects carvedilol were assessed utilizing a system of clinical sign scores, and an enzyme-linked immunosorbent assay was used to determine the expression levels of interleukin-1 $\beta$ (IL-1 $\beta$ ), IL-6, IL- 8 and tumor necrosis factor- $\alpha$ (TNF- $\alpha$ ). Finally, nuclear factor- $\kappa \mathrm{B}$ $(\mathrm{NF}-\kappa \mathrm{B})$, nerve growth factor (NGF) and vascular endothelial growth factor (VEGF) were analyzed by western blotting. Carvedilol was observed to significantly reduce clinical sign scores in a dose-dependent manner $(\mathrm{P}<0.01)$, and reduce IL-1 $\beta$, IL-6, IL- 8 and TNF- $\alpha$ expression levels $(\mathrm{P}<0.01)$ in the LPS-induced rat model of conjunctivitis. Carvedilol was also able to significantly reduce the protein expression levels of $\mathrm{NF}-\kappa \mathrm{B}$, and induce the protein expression levels of NGF and VEGF in the LPS-induced rat model of conjunctivitis $(\mathrm{P}<0.01)$. In conclusion, the effects of carvedilol may reduce conjunctivitis clinical scores through inflammation, NGF and VEGF in LPS-induced rat models.
\end{abstract}

Correspondence to: Ms. Ying Chen, Department of Ophthalmology, Jinhua Traditional Chinese Medical Hospital, 439 Shuangxi West Road, Jinhua, Zhejiang 321000, P.R. China E-mail: yingchenchenzd@yeah.net

Key words: carvedilol, conjunctivitis, inflammation, nerve growth factor, vascular endothelial growth factor

\section{Introduction}

Conjunctivitis is the most common allergic eye disease and the most frequently observed ocular surface disease in China (1). According to statistics, $5 \%$ of the global population have sought medical attention due to an allergic eye disease, $50 \%$ of which were cases of conjunctivitis (1). Recently, in part as a result of the increased use of eye makeup and the increase of air pollution in certain regions, the incidence of conjunctivitis has increased (2).

Conjunctiva is a transparent layer of mucosal tissue, rich in nerves and blood vessels, that is important for eye protection and lubrication, serving as the protective film of the eye (3). Conjunctivitis can be classified as infectious or non-infectious, with infection taking precedence as the most common cause (4). Pathogenic microorganisms, including bacteria (such as chlamydia, rickettsia), viruses, fungi and parasites (5), are typically transmitted through air, water, physical contact or via tissue adjacent to the affected area by inflammation (including the cornea, eyelid and lacrimal apparatus) (6). Non-infectious conjunctivitis is typically a result of physical stimuli (namely dust mites, smoke, dust and ultraviolet light) or chemical stimuli (including poisonous gas, medical drugs, medical drugs, cosmetics and shampoos) (7).

Although chronic conjunctivitis displays subjective symptoms and involves a characteristically slow disease progression compared with acute conjunctivitis, relapse is common (8). Upon further inspection mild conjunctival congestion edema, papillary hyperplasia, a small amount of white mucous or secretions, such as foam samples may be observed; if not treated promptly, chronic conjunctivitis may develop into carcinomas able to infiltrate the cornea and affect vision (9). Therefore, chronic conjunctivitis requires attention, and further investigation should be performed to identify its causes, eliminate any responsible pathogenic factors, and develop the necessary targeted therapies (10).

Carvedilol is a novel non-selective adrenergic receptor blocker that displays no inherent sympathetic activity. Therefore, the drug bypasses the reflexive excitement of the nervous system often caused by peripheral vascular contraction and increased peripheral resistance. In addition, carvedilol is an oxygen free-radical scavenger that regulates cell factors, such as interleukins and colony stimulating factors, and is involved in a variety of actions $(11,12)$. However, the effect of carvedilol on conjunctivitis has rarely been reported. 
Accordingly, the present study aimed to evaluate whether the known effects of carvedilol are able to reduce conjunctivitis clinical scores.

\section{Materials and methods}

Animals and modeling. Adult Sprague Dawley (SD) rats ( $\mathrm{n}=24$; purchased from the Animal Experimental Center of Zhejiang University, Zhejiang, China) weighing 230-300 g were housed in a 12-h light/dark cycle at a temperature of $22-26^{\circ} \mathrm{C}$, and were provided with food and water ad libitum. The study was approved by the ethics committee of Zhejiang University School of Medicine (Huangzhou, China). All SD rats were anesthetized with $75 \mathrm{mg} / \mathrm{kg}$ ketamine (injectable IMALGENE 1000; Merial, Lyon, France) and $10 \mathrm{mg} / \mathrm{kg}$ xylazine (Rompun 2\%; Beyotime Institute of Biotechnology, Haimen, China) by intraperitoneal injection (i.p.). Next, each eye of the anesthetized rats was subconjunctivally injected with $30 \mu 1$ lipopolysaccharide (LPS; Sigma-Aldrich Química S.A., Tres Cantos, Spain). Further treatment of the rats with LPS-induced conjunctivitis commenced after $24 \mathrm{~h}$.

Study groups. SD rats were randomly divided into three equal groups: i) Control group, consisting of eight SD rats treated with $1 \mathrm{ml} 0.9 \% \mathrm{NaCl}$ (i.p.) for 4 weeks (i.p.); ii) a model group, consisting of eight LPS-induced conjunctivitis rats administered with $1 \mathrm{ml} 0.9 \% \mathrm{NaCl}$ (i.p.) for 4 weeks; and iii) the carvedilol treatment group, consisting of eight LPS-induced conjunctivitis rats treated with $50 \mathrm{mg} / \mathrm{kg}$ /day carvedilol (i.p.; Sigma-Aldrich, St. Louis, MO, USA) for 4 weeks.

Evaluation of clinical sign scores. Prior to the euthanasia of the SD rats, clinical sign scores were graded by three blind independent evaluators, as previously reported (13). The clinical score signs were recorded prior to induction of conjunctivitis, and $1,2,3$ and 4 weeks after carvedilol administration. Clinical sign scores were obtained by three blind independent observers according to previously described criteria (14).

Evaluation of interleukin (IL)-1 $\beta, I L-6, I L-8$ and tumor necrosis factor- $\alpha(T N F-\alpha)$ with enzyme-linked immunosorbent assay (ELISA). After 4 weeks of treatment with carvedilol, rats were sacrificed by cervical dislocation. Then, peripheral blood was collected from the eye sockets of the rats. Blood samples were centrifuged at $3,000 \mathrm{x}$ g for $20 \mathrm{~min}$, and the supernatant was collected and stored at $-80^{\circ} \mathrm{C}$ for further assessment. IL-1 $\beta$ (m1028611), IL-6 (m1002828), IL-8 (m1027376) and TNF- $\alpha$ (m1002859) expression levels were measured using ELISA, according to the manufacturer's protocol (Shanghai Boya Biotechnology Co., Ltd., Shanghai, China).

Evaluation of nuclear factor- $\kappa B(N F-\kappa B)$, nerve growth factor (NGF) and vascular endothelial growth factor (VEGF) expression levels with western blotting. After 4 weeks of treatment with carvedilol, rats were sacrificed by cervical dislocation. Then, conjunctivitis tissue samples were collected and homogenized in modified RIPA buffer (pH 7.4; Beyotime Institute of Biotechnology). Cytosolic protein samples were centrifuged at $12,000 \mathrm{x} \mathrm{g}$ for $10 \mathrm{~min}$ at $4^{\circ} \mathrm{C}$ and the supernatant was collected. Protein concentration was determined

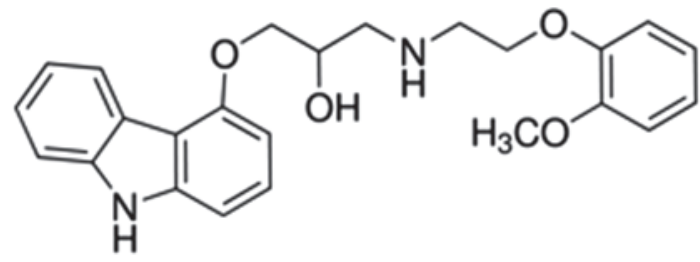

Figure 1. Chemical structure of carvedilol.

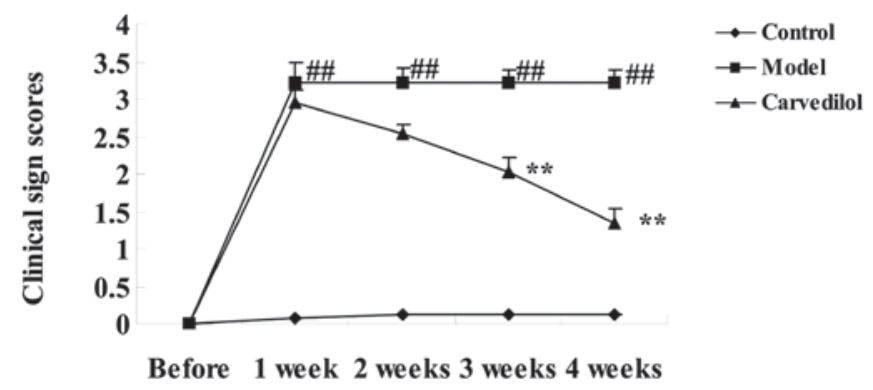

Figure 2. Effects of carvedilol on clinical sign scores in rat models of conjunctivitis. ${ }^{\# \#} \mathrm{P}<0.01$ vs. control group; ${ }^{* *} \mathrm{P}<0.01$ vs. model group. Data are presented as mean \pm standard deviation.

using a BCA assay kit (Shanghai Boya Biotechnology Co., Ltd.) and equal quantities of protein were separated by $10 \%$ sodium dodecyl sulfate-polyacrylamide gel electrophoresis at room temperature, then transferred by electroblotting to nitrocellulose membranes (Hybond-C Extra; GE Healthcare Life Sciences, Pittsburgh, PA, USA). The nitrocellulose membranes were blocked in blocking buffer (Tris-buffered saline and $0.1 \%$ Tween-20; Beijing Biosntech Biotechnology Co., Ltd., Beijing, China) containing 5\% skim milk, incubated with polyclonal rabbit anti-NF-kB (1:500; sc-7151), polyclonal rabbit anti-NGF $(1: 2,000 ;$ sc-33602) and polyclonal rabbit anti-VEGF (1:1,000; sc-507) (all purchased from Santa Cruz Biotechnology Inc., Dallas, TX, USA) antibodies, washed and then incubated with horseradish peroxidase-conjugated secondary antibody (1:3,000; sc-45101; Santa Cruz Biotechnology Inc.). The resultant bands were visualized by chemiluminescent detection (ECL Western Blotting Detection Reagent; GE Healthcare, Chalfont, UK) and analyzed using a computer imaging system (GDS-8000 Image Acquisition and Analysis System; UVP, LLC, Upland, CA, USA). The reactions were repeated 3 times.

Statistical analysis. All results are expressed as the mean \pm standard deviation, and analysis was performed using two-way analysis of variance on the computer program SPSS (version 18.0; SPSS, Inc., Chicago, IL, USA). A value of $\mathrm{P}<0.05$ was considered to indicate a statistically significant difference.

\section{Results}

Effects of carvedilol on clinical sign scores in conjunctivitis rat models. The chemical structure of carvedilol is displayed in Fig. 1. The effects of carvedilol on clinical sign scores was examined. LPS significantly increased clinical sign scores in the conjunctivitis rat model group compared with the control group $(\mathrm{P}<0.01)$. By contrast, carvedilol significantly blocked 
A

$$
\text { C }
$$

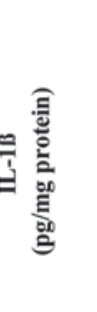

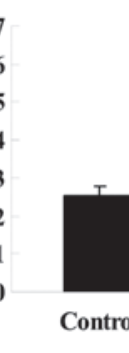

衰

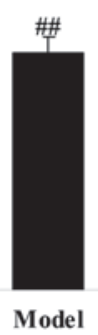

Model
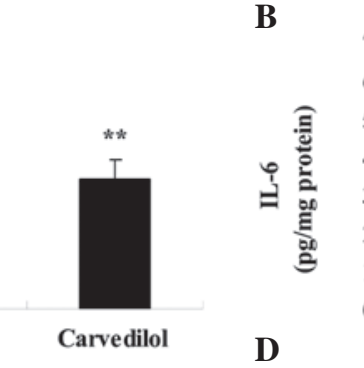
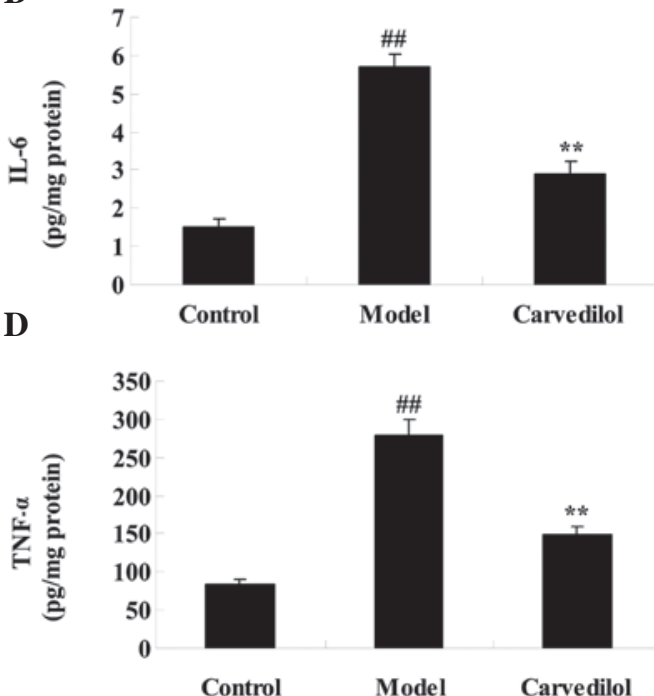

Mode

Carvedilol
350

300

100

50

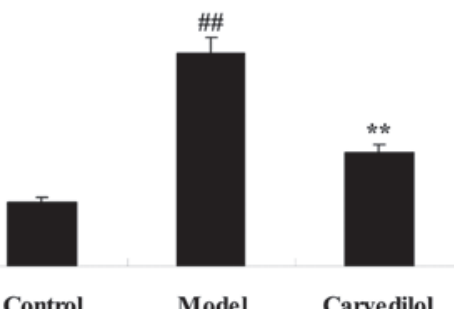

Figure 3. Effects of carvedilol on (A) IL-1 $\beta$ (B) IL-6 (C) IL-8 and (D) TNF- $\alpha$ in a rat model of conjunctivitis. ${ }^{\# \#} \mathrm{P}<0.01$ vs. control group; ${ }^{* *} \mathrm{P}<0.01 \mathrm{vs.} \mathrm{model}$ group. Data are presented as mean \pm standard deviation. IL, interleukin; TNF- $\alpha$, tumor necrosis factor- $\alpha$.

A

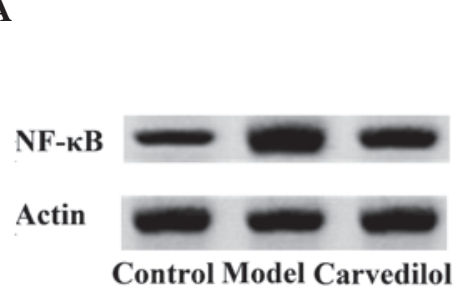

B

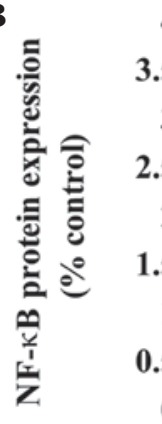

4

3.5

3

2.5

1.5

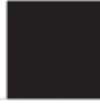

Control 
A

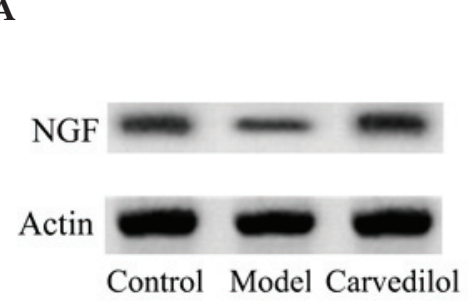

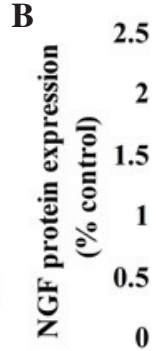

Control

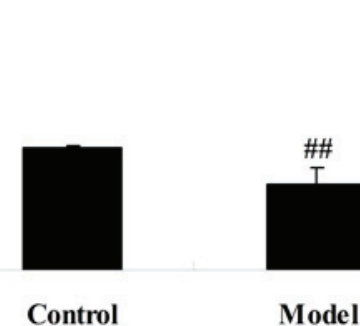

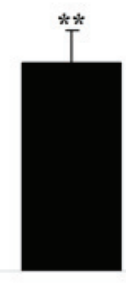

Carvedilol

Figure 5. Effects of carvedilol on NGF protein expression levels in a rat model of conjunctivitis using (A) western blotting assays and (B) statistical analysis. ${ }^{\# \#} \mathrm{P}<0.01$ vs. control group; ${ }^{* *} \mathrm{P}<0.01$ vs. model group. Data are presented as mean \pm standard deviation. NGF, nerve growth factor.

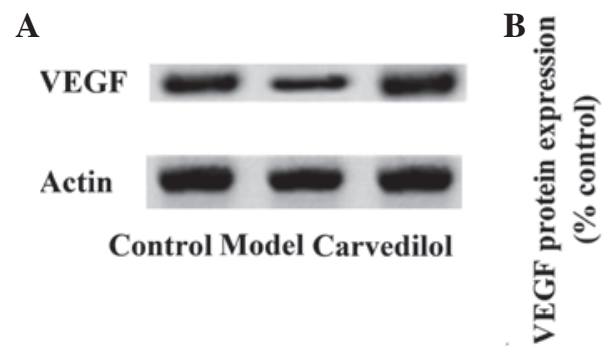

1.5

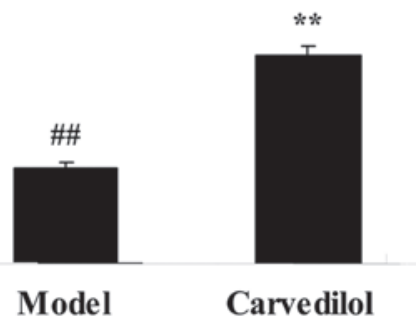

Figure 6. Effects of carvedilol on VEGF protein expression levels in a rat model of conjunctivitis using (A) western blotting assays and (B) statistical analysis. ${ }^{\#} \mathrm{P}<0.01$ vs. control group; ${ }^{* *} \mathrm{P}<0.01$ vs. model group. Data are presented as mean \pm standard deviation. VEGF, vascular endothelial growth factor.

were significantly reduced in the conjunctivitis model group compared with the carvedilol group ( $\mathrm{P}<0.01$; Fig. 6).

\section{Discussion}

Conjunctivitis is a frequently observed allergic eye disease. In total, $\sim 5 \%$ of the population have sought medical advice as a result of an allergic eye disease, of which $>50 \%$ were cases of conjunctivitis (15). In recent years, due to increases in factors such as air pollution and the use of contact lenses, the incidence of conjunctivitis has risen (16). Patients typically present with itchiness of the eye, photophobia, lacrimation and a foreign body or burning sensation. Although the majority of conjunctivitis cases rarely result in severe complications, repeated incidence of the disease are common, and cause severe eye discomfort and itching, which may affect the patient's quality of life and work efficiency by reducing their ability to concentrate (17). To the best of our knowledge, the present study revealed for the first time that carvedilol significantly reduces augmented clinical sign scores in a rat model of conjunctivitis.

A recent study revealed that conjunctival infection can cause systemic inflammatory responses involving the induction of inflammatory cytokines, including IL-1 $\beta$, IL- 6 , IL-8 and TNF- $\alpha$ (18). It was previously identified that IL-1 $\beta$ is an important inflammatory cytokine, and participates in inflammatory responses to injury and autoimmune diseases. IL-1 $\beta$, IL-6, IL-8, TNF- $\alpha$ and neutrophils are also involved in an autocrine loop of inflammatory mediators (19). Patients commonly develop with acute conjunctivitis as a result of bacterial or viral infections, which trigger inflammation, and cause mononuclear cells to produce high levels of IL-1 $\beta$, IL-6, IL-8 and TNF- $\alpha$. This results in elevated levels of the aforementioned inflammatory cytokines in the blood plasma. Furthermore, cell response function is reduced in acute conjunctivitis, resulting in the disruption of the cellular immune network. In particular, disorders may occur in the percentage of $\mathrm{T}$ and $\mathrm{B}$ lymphocytes, resulting in increased plasma expression levels of inflammatory cytokines (20). In the present study, elevated IL-1 $\beta$, IL-6, IL-8, TNF- $\alpha$ and NF- $\kappa B$ expression levels were revealed to be significantly suppressed following treatment with carvedilol. Similarly, de Araújo Júnior et al demonstrated that carvedilol decreased IL-1 $\beta$ and TNF- $\alpha$ expression levels in a rat model of periodontitis (21). Additionally, Arab and El-Sawalhi reported that carvedilol alleviated adjuvant-induced arthritis inflammatory mediators (22). Thus, the effect of carvedilol on conjunctivitis may involve the suppression of inflammatory mediators in rats.

Conjunctivitis is a complex inflammatory response involving a variety of factors. Several non-immune factors, including nerve- and endocrine-related factors, may affect the pathological changes and clinical manifestations of conjunctivitis (23). In the serum of patients with conjunctivitis, NGF expression increases significantly; this appears to be associated with the infiltration of mast cells in the palpebral conjunctiva (24). Patients with conjunctivitis also displayed increased levels of neuropeptide, which are synthesized and released by various cell factors involved in NGF regulation and control. Thus, the increased expression levels of NGF appear to result in increased neuropeptide expression in patients with conjunctivitis (25). The phenomenon of increased NGF expression levels is not exclusive to conjunctivitis but also exists in other systemic allergic diseases (26). In the present study, it was revealed that carvedilol significantly increases the suppression of NGF protein expression levels in rat models 
of conjunctivitis. Shyu et al revealed that NGF mRNA and protein expression levels were upregulated by carvedilol in pressure-overloaded rat hearts (27) and a rat model of volume-overload heart failure (28). These results indicate that the effect of carvedilol on inflammatory mediators may serve to upregulate NGF signaling.

It has been revealed that epithelial cells, inflammatory cells (eosinophils, monocytes/macrophages) and conjunctival fibroblasts produce VEGF following stimulation (29). In angiogenesis, VEGF in epithelial cells serves a core role in vascular matrix change (30). Fibroblasts are another source of the VEGF (31). In the present study, it was demonstrated that carvedilol significantly increases VEGF protein expression levels in rat models of conjunctivitis. Similarly, de Boer et al previously reported that carvedilol increased VEGF expression levels in patients with chronic heart failure (32). The results indicate that the effect of carvedilol on conjunctivitis may involve the upregulation of VEGF.

In conclusion, carvedilol is able to reduce the symptoms of conjunctivitis in rat models, and its effect may be associated with the reversal of the abnormal regulation of inflammation, as well as increased NGF and VEGF expression levels, in rats with conjunctivitis. Carvedilol may, therefore, be a potential novel therapy for conjunctivitis.

\section{Acknowledgements}

The present study was supported by the Zhejiang Provincial Hospital of Traditional Chinese Medicine (Hangzhou, China; grant no. 2014ZA118).

\section{References}

1. Saha S, Koley M, Ghosh S, Giri M, Das A and Goenka R: Documentation of prescriptions and clinical outcomes in a homeopathic hospital setting in West Bengal, India. J Evid Based Complementary Altern Med 20:180-185, 2015.

2. Schwartz B, Harrison LH, Motter JS, Motter RN, Hightower AW and Broome CV: Investigation of an outbreak of Moraxella conjunctivitis at a Navajo boarding school. Am J Ophthalmol 107: 341-347, 1989.

3. Chen Y, Zhang X, Yang L, Li M, Li B, Wang W and Sheng M: Decreased PPAR- $\gamma$ expression in the conjunctiva and increased expression of TNF- $\alpha$ and IL-1 $\beta$ in the conjunctiva and tear fluid of dry eye mice. Mol Med Rep 9: 2015-2023, 2014.

4. Barisani-Asenbauer T, Inic-Kanada A, Belij S, Marinkovic E, Stojicevic I, Montanaro J, Stein E, Bintner N and Stojanovic M The ocular conjunctiva as a mucosal immunization route: A profile of the immune response to the model antigen tetanus toxoid. PLoS One 8: e60682, 2013.

5. Koulikovska M, van der Ploeg I, Herrmann B and Montan PG: Respiratory syncytial virus and chlamydia are not detectable by PCR in ongoing vernal keratoconjunctivitis. Ocul Immunol Inflamm 9: 253-257, 2001.

6. Grayston JT, Woolridge RL, Chen CW, Assaad FA, Maffei S, Yen $\mathrm{CH}$ and Yang CY: Bacterial conjunctivitis caused by a base eye ointment used as a placebo. Rep U S Nav Med Res Lab 4: 1-11, 1960.

7. Doughty MJ: Sodium cromoglycate ophthalmic solution as a Pharmacy Medicine for the management of mild-to-moderate, non-infectious inflammation of the conjunctiva in adults. Ophthalmic Physiol Opt 16 (Suppl 2): S33-S38, 1996.

8. Buckley P and Lowman DM: Chronic non-infective conjunctivitis in rabbits. Lab Anim 13: 69-73, 1979.

9. Nell B, Walde I, Billich A, Vit P and Meingassner JG: The effect of topical pimecrolimus on keratoconjunctivitis sicca and chronic superficial keratitis in dogs: Results from an exploratory study. Vet Ophthalmol 8: 39-46, 2005.
10. Ferreri AJ, Dolcetti R, Du MQ, Doglioni C, Resti AG, Politi LS, De Conciliis C, Radford J, Bertoni F, Zucca E, et al: Ocular adnexal MALT lymphoma: An intriguing model for antigen-driven lymphomagenesis and microbial-targeted therapy. Ann Oncol 19: 835-846, 2008.

11. Chen J, Huang C, Zhang B, Huang Q, Chen J and Xu L: The effects of carvedilol on cardiac structural remodeling: The role of endogenous nitric oxide in the activity of carvedilol. Mol Med Rep 7: 1155-1158, 2013.

12. Ronsein GE, Guidi DB, Benassi JC, Filho DW, Pedrosa RC and Pedrosa RC: Cytoprotective effects of carvedilol against oxygen free radical generation in rat liver. Redox Rep 10: 131-137, 2005.

13. Merayo-Lloves J, Zhao TZ, Dutt JE and Foster CS: A new murine model of allergic conjunctivitis and effectiveness of nedocromil sodium. J Allergy Clin Immunol 97: 1129-1140, 1996.

14. Gong L, Sun X, Qu J, Wang L, Zhang M, Zhang H, Wang L, $\mathrm{Gu} \mathrm{Y}$, Elion-Mboussa A, Roy L and Zhu B: Loteprednol etabonate suspension $0.2 \%$ administered QID compared with olopatadine solution $0.1 \%$ administered BID in the treatment of seasonal allergic conjunctivitis: A multicenter, randomized, investigator-masked, parallel group study in Chinese patients. Clin Ther 34: 1259-1272, 2012.

15. Chansaenroj J, Vongpunsawad S, Puenpa J, Theamboonlers A, Vuthitanachot V, ChattakulP, Areechokchai D and Poovorawan Y: Epidemic outbreak of acute haemorrhagic conjunctivitis caused by coxsackievirus A24 in Thailand, 2014. Epidemiol Infect 143: 3087-3093, 2015

16. Chang CJ, Yang HH, Chang CA and Tsai HY: Relationship between air pollution and outpatient visits for nonspecific conjunctivitis. Invest Ophthalmol Vis Sci 53: 429-433, 2012.

17. Yasin MN, Hussain S, Malik F, Hameed A, Sultan T, Qureshi F, Riaz H, Perveen G and Wajid A: Preparation and characterization of chloramphenicol niosomes and comparison with chloramphenicol eye drops $(0.5 \% \mathrm{w} / \mathrm{v})$ in experimental conjunctivitis in albino rabbits. Pak J Pharm Sci 25: 117-121, 2012.

18. Tanaka J, Tanaka H, Mizuki N, Nomura E, Ito N, Nomura N, Yamane M, Hida T, Goshima Y, Hatano H, et al: Semaphorin $3 \mathrm{~A}$ controls allergic and inflammatory responses in experimental allergic conjunctivitis. Int J Ophthalmol 8: 1-10, 2015.

19. Enríquez-de-Salamanca A, Calder V, Gao J, Galatowicz G, García-Vázquez C, Fernández I, Stern ME, Diebold Y and Calonge M: Cytokine responses by conjunctival epithelial cells: An in vitro model of ocular inflammation. Cytokine 44: 160-167, 2008.

20. Leonardi A, Curnow SJ, Zhan H and Calder VL: Multiple cytokines in human tear specimens in seasonal and chronic allergic eye disease and in conjunctival fibroblast cultures. Clin Exp Allergy 36: 777-784, 2006.

21. de Araújo Júnior RF, Souza TO, de Medeiros CA, de Souza LB, Freitas ML, de Lucena HF, do Socorro Costa Feitosa Alves M and de Araújo AA: Carvedilol decrease IL-1 $\beta$ and TNF- $\alpha$, inhibits MMP-2, MMP-9, COX-2, and RANKL expression, and up-regulates OPG in a rat model of periodontitis. PLoS One 8: e66391, 2013.

22. Arab HH and El-Sawalhi MM: Carvedilol alleviates adjuvant-induced arthritis and subcutaneous air pouch edema: Modulation of oxidative stress and inflammatory mediators. Toxicol Appl Pharmacol 268: 241-248, 2013.

23. Levi-Schaffer F, Micera A, Zamir E, Mechoulam H, Puxeddu I, Piliponsky AM, Aloe L and Pe'er J: Nerve growth factor and eosinophils in inflamed juvenile conjunctival nevus. Invest Ophthalmol Vis Sci 43: 1850-1856, 2002.

24. Lambiase A, Bonini S, Micera A, Rama P, Bonini S and Aloe L Expression of nerve growth factor receptors on the ocular surface in healthy subjects and during manifestation of inflammatory diseases. Invest Ophthalmol Vis Sci 39: 1272-1275, 1998.

25. Kritas SK, Saggini A, Cerulli G, Caraffa A, Antinolfi P, Pantalone A, Frydas S, Rosati M, Tei M, Speziali A, et al: Neuropeptide NGF mediates neuro-immune response and inflammation through mast cell activation. J Biol Regul Homeost Agents 28: 177-181, 2014.

26. Micera A, Stampachiacchiere B, Normando EM, Lambiase A, Bonini S and Bonini S: Nerve growth factor modulates toll-like receptor (TLR) 4 and 9 expression in cultured primary VKC conjunctival epithelial cells. Mol Vis 15: 2037-2044, 2009.

27. Shyu KG, Liou JY, Wang BW, Fang WJ and Chang H: Carvedilol prevents cardiac hypertrophy and overexpression of hypoxia-inducible factor-1alpha and vascular endothelial growth factor in pressure-overloaded rat heart. J Biomed Sci 12: 409-420, 2005 
28. Shyu KG, Lu MJ, Chang H, Sun HY, Wang BW and Kuan P: Carvedilol modulates the expression of hypoxia-inducible factor-1alpha and vascular endothelial growth factor in a rat model of volume-overload heart failure. J Card Fail 11: 152-159, 2005.

29. Wang F, Xu P, Xie KC, Chen XF, Li CY and Huang Q: Effects of tumor microenviromental factors on VEGF expression. Biomed Rep 1: 539-544, 2013.

30. Taurone S, Ripandelli G, Pacella E, Bianchi E, Plateroti AM, De Vito S, Plateroti P, Grippaudo FR, Cavallotti C and Artico M: Potential regulatory molecules in the human trabecular meshwork of patients with glaucoma: Immunohistochemical profile of a number of inflammatory cytokines. Mol Med Rep 11: 1384-1390, 2015.
31. Goi T, Nakazawa T, Hirono Y and Yamaguchi A: The anti-tumor effect is enhanced by simultaneously targeting VEGF and PROK1 in colorectal cancer. Oncotarget 8: 6053-6061, 2015 .

32. de Boer RA, Siebelink HJ, Tio RA, Boomsma F and van Veldhuisen DJ: Carvedilol increases plasma vascular endothelial growth factor (VEGF) in patients with chronic heart failure. Eur J Heart Fail 3: 331-333, 2001. 